\title{
Evaluation of the serum visfatin eotaxin and fetuin-A levels of patients with type 2 diabetes mellitus
}

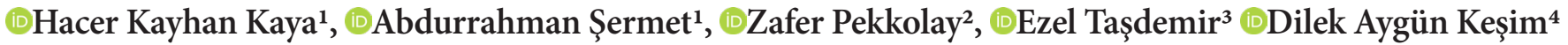 \\ ${ }^{1}$ Dicle University, Faculty of Medicine, Department of Physiology, Diyarbakır, Turkey \\ ${ }^{2}$ Dicle University, Department of Internal Medicine, Diyarbakır, Turkey \\ ${ }^{3}$ Medicalpark Hospitals, Department of Internal Medicine,Antalya, Turkey \\ ${ }^{4}$ Dicle University, Faculty of Medicine, Department of Physical Medicine and Rehabilitation, Diyarbakır, Turkey
}

Cite this article as: Kayhan Kaya H, Şermet A, Pekkolay Z, Taşdemir E, Aygün Keşim D. Evaluation of the serum visfatin eotaxin and fetuin-A levels of patients with type 2 diabetes mellitus. Anatolian Curr Med J 2022; 4(1); 113-119.

\begin{abstract}
Objective: The aim of this study was to determine the serum visfatin, eotaxin and fetuin-A levels in patients with normal BMI and overweight type 2 diabetes mellitus (T2DM).

Material and Method: This study perform in 30 T2DM patients and in 20 healthy subjects. Test subjects were divided into four groups as two diabetics and two controls. Diabetics with a body mass index (BMI) of $26.2-29.9 \mathrm{~kg} / \mathrm{m}^{2}$ were included in the overweight diabetic group (OD), and those with a body mass index of $20.9-24.9 \mathrm{~kg} / \mathrm{m}^{2}$ were included in the normal BMI diabetic group (ND). The volunteers in the control group were also divided into two groups as overweight (OC) and normal BMI (NC). Smoking and alcohol users were not included in the study. In addition, patients with significant diabetic complications such as retinopathy, hypertension, neuropathy, renal failure, and cardiovascular disease were excluded from the study. The serum visfatin eotaxin and fetuin-A levels were measured using the ELISA method. The Mann-Whitney U test was used to compare the data of the groups, while Spearman's analysis was applied for the correlations.

Results: The visfatin levels of the OD and ND were significantly higher compared to those of their control groups ( $\mathrm{p}<0.05$ and $\mathrm{p}<0.05$ respectively). In addition, the eotaxin levels of the diabetic patients both OD and ND were significantly higher than those of their control group $(\mathrm{p}<0.001$ and $\mathrm{p}<0.05$, respectively). Serum fetuin-A level was not different between groups.

Conclusion: Serum visfatin and eotaxin levels are high in patients with T2DM, and this elevation is dependent on BMI. In addition, visfatin level is high in overweight non-diabetic subjects.
\end{abstract}

Keywords: Visfatin, eotaxin, fetuin-A, type 2 diabetes mellitus.

\section{INTRODUCTION}

Type 2 diabetes mellitus (T2DM) is accepted as one of the most prominent metabolic diseases of the 21 st century (1). The main reason for this disease is insulin resistance, increased liver glucose production, and deficiency in insulin secretion against glucose stimulation $(2,3)$. Although the pathogenesis of T2DM is not completely known, it is thought to be due to the interactions of genetic, environmental, and behavioral risk factors (4). Economic developments, urbanization, longevity, physical inactivity, unhealthy diet, smoking, drinking alcohol, and obesity all contribute to the development of T2DM (5). A significant number of patients with T2DM are obese and the incidence of diabetes is closely related to the increase in obesity prevalence (6). Obesity to the development of the disease in approximately $55 \%$ of patients with T2DM (7).
A parallel relation is thought to exist between the pancreas and adipose tissue, and the majority of patients with T2DM are obese individuals with visceral fat. Overweight and obesity causes an increase in adipose tissue stores and irregular adipokine secretion. Adipokines secreted from macrophages that infiltrate fat cells and adipose tissue are believed to cause a low-grade chronic inflammatory condition, which in turn, leads to the insulin resistance in tissues such as liver and skeletal muscle, and subsequently to T2DM (8).

Visfatin, which is a new member of the adipocytokine family, was first described by Fukuhara et al. (9) in 2005. The most interesting feature of visfatin is its insulin-mimetic feature, which has only recently been put forward. It has been reported that visfatin mimics the effects of insulin such as inhibiting hepatic glucose 
release, increasing glucose uptake in fat and muscle cells, and increasing triglyceride synthesis. It has been found that visfatin activates the phosphorylation and signal transmission of insulin receptors by binding to a place that is different to the place where insulin binds and using a different pathway to insulin (9).

It is known that chronic inflammation plays a part in the pathogenesis of T2DM and that various cytokines play a critical role in the onset and progress of the disease. Eotaxin is a fundamental cytokine in the pathogenesis of allergic respiratory diseases, inflammatory bowel disease and gastrointestinal allergic hypersensitivity (10). It has been reported that eotaxin is a secretory product of adipose tissue and its plasma level increased in obese patients and is believed to be associated with the pathogenesis of diabetes (11).

It has been determined that protein structured fetuin-A synthesized in the liver significantly increases with obesity. Moreover, it has been suggested that there is a significant link between obesity and fetuin- $A$ and insulin resistance in humans $(12,13)$. Recent researches have reported that fetuin-A can play a part in the glucose metabolism and induce insulin resistance in target tissues by inhibiting insulin receptor activity in the liver, muscles and adipose tissue $(14,15)$. Within this context, in this study determined the visfatin, eotaxin and fetuin- $A$ levels of normal and overweight patients with T2DM and investigated their relationship with fasting blood glucose (FBG), insulin resistance, fasting insulin level, BMI, and the relationship between these molecules.

\section{MATERIAL AND METHOD}

The study was approved by the Non-interventional Studies Ethics Committee of Dicle University Faculty of Medicine (Date: 15.04.2015, Decision No: 195). Consent was acquired from all of the participants, who were also informed about what to do, also the study was carried out in accordance with the Declaration of Helsinki.

\section{Subjects}

The study was comprised of 30 patients who had applied to the Endocrinology and Metabolic Diseases Outpatient Clinic of Dicle University Hospital, had been diagnosed with T2DM in accordance with the American Diabetes Association (ADA) criteria, had received oral antidiabetic treatment and were between the ages of 47 63 years and 20 healthy participants between the same ages. The patients and healthy participants included in the study were selected from people who did not smoke or drink alcohol. In addition, patients with significant diabetic complications such as retinopathy, hypertension, neuropathy, renal failure, and cardiovascular disease were excluded from the experiment. The BMI of the participants was calculated when they were first admitted. The 30 patients with T2DM and healty participants were divided into two groups according to their BMI. Those with a BMI of $26.2-29.9 \mathrm{~kg} / \mathrm{m}^{2}$ were included in the overweight diabetic group, while those with a BMI between $20.9-24.9 \mathrm{~kg} / \mathrm{m}^{2}$ were included in the normalweight diabetic group. The volunteer subjects in the control group were also divided into two groups in the same way. All patients were using metformin at a dose of $2000 \mathrm{mg} /$ day for more than 1 year.

\section{Blood Sampling and Measurements}

The biochemical measurements of the participants were carried out at the Central Laboratory of Dicle University Hospital. In venous blood samples were obtained from the participants after at least 8-12 hours of fasting to determine their glucose measurement. These measurements were carried out with Abbott Diagnostics original kits and in accordance with the spectrophotometrical method. The glycated hemoglobin (HbAlc) levels of the participants were also measured on the same day according to the liquid chromatography (HPLC) method and the fasting insulin levels were determined by using Roche Diagnostics original kits and the Cobas e601 module (Roche Diagnostics, Mannheim, Germany) in accordance with the electrochemiluminescence measurement method. An extra tube of blood was taken from the participants and centrifuged at $4000 \mathrm{rpm}$ for ten minutes then the serum samples were taken into another eppendorf tube. The visfatin, eotaxin and fetuin-A levels in the serum samples stored at $-80^{\circ} \mathrm{C}$ were measured in accordance with the ELISA method using human ELISA kits (visfatin catalog number: 201-12-0026; SunRed Biotechnology, Shanghai, China, eotaxin (catalog number: CK-E90892; Hangzhou Eastbiopharm Co., Ltd., Zhejiang, China, and fetuin-A catalog number: 201-12-1387; SunRed Biotechnology, Shanghai, China). The insulin resistance of the patient and control groups were calculated according to the HOMA-IR index (16).

\section{Statistical Analysis}

Microsoft Excel was used to record the data collected from the all participants, and the statistical analyses were carried out using SPSS software (version 18.0). Kolmogorov-Smirnov test was used for checking normality of the obtained data. Since the data were not normally distributed, The Mann Whitney $U$ test was used for the analysis of the data. Moreover, to investigate the correlation of visfatin, eotaxin and fetuin-A with FBG, HbAlc, fasting insulin, Insulin resistance, and BMI Spearman's correlation analysis was applied. The results were stated as median values. Furthermore, $p$ values less than $5 \%(\mathrm{p}<0.05)$ were considered significant, while those greater than $5 \%(p>0.05)$ were considered insignificant. 


\section{RESULTS}

The principal characteristics of all groups are presented in Table 1. The patient groups and their control groups were found to be similar in terms of age, height, weight, and BMI.

The FBG level of the OD was measured as $137 \mathrm{mg} / \mathrm{dl}$, while the level of the ND was found as $140 \mathrm{mg} / \mathrm{dl}$. Even though both patient groups were using oral antidiabetic medicines their FBG levels were found to be significantly higher than those of their control groups (OD: $p<0.001$ and ND: $\mathrm{p}<0.05)$. The fasting insulin level of the OD was found to be significantly higher than the ND $(\mathrm{p}<0.05)$. The $\mathrm{HbAlc}$ values were significantly higher in the $\mathrm{OD}$ and ND compared to their control groups $(\mathrm{P}<0.001)$. Insulin resistance was significantly higher in the $\mathrm{OD}$ compared to the ND $(\mathrm{p}<0.05)$.

The visfatin level of the OD was found to be $16.65 \mathrm{ng} / \mathrm{ml}$, while those of the OC was $10.60 \mathrm{ng} / \mathrm{ml}$, those of the ND was $14 \mathrm{ng} / \mathrm{ml}$ and those of the NC was $3.50 \mathrm{ng} / \mathrm{ml}$. The visfatin levels of the OD and ND were significantly higher than those in the control groups as shown in Figure 1 $(p<0.05)$. Similarly, the eotaxin levels were found to

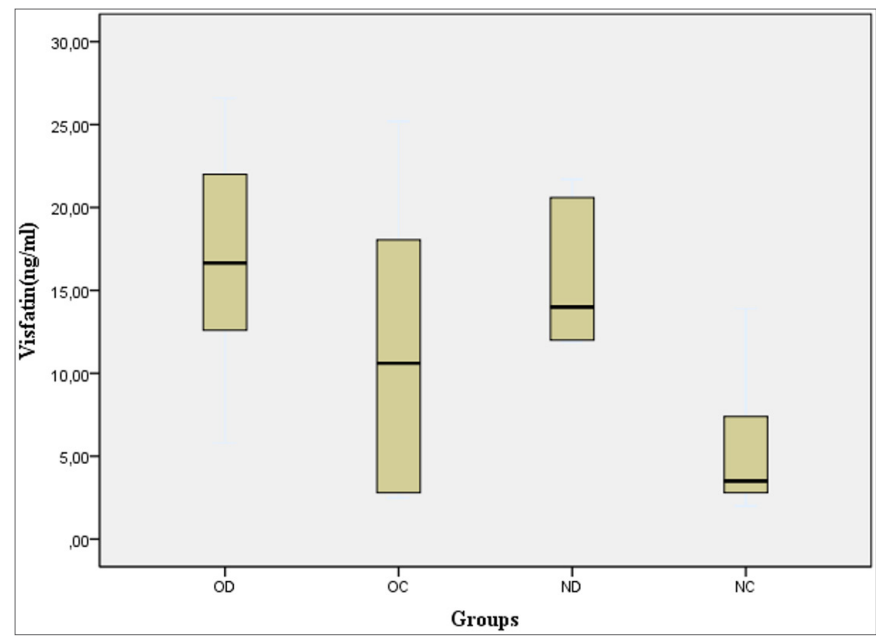

Figure 1. A Boxplot illustration of serum visfatin concentrations in subjects of overweight and normalweight diabetic patients and their healty controls. OD: overweight diabetic, OC: overweight control, ND: normalweight diabetic, NC: normalweight control be significantly higher in the OD and ND compared to the control groups as shown in Figure 2 ( $p<0.001$ and $\mathrm{p}<0.05)$. No significant difference was determined between the fetuin-A levels of OD and ND and those of the controls (Figure 3).

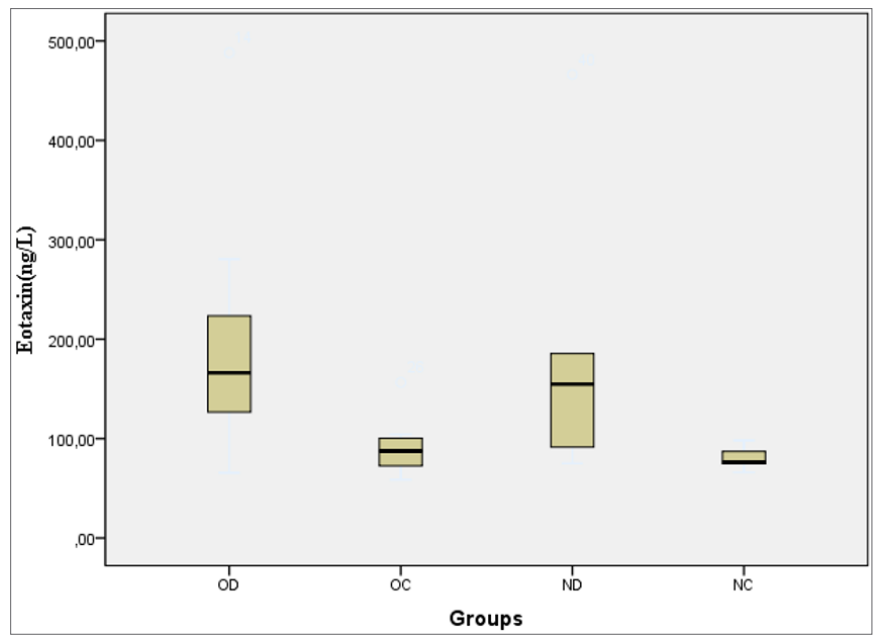

Figure 2. A Boxplot illustration of serum eotaxin concentrations in subjects of overweight and normalweight diabetic patients and their healty controls. OD: overweight diabetic, OC: overweight control, ND: normalweight diabetic, NC: normalweight control

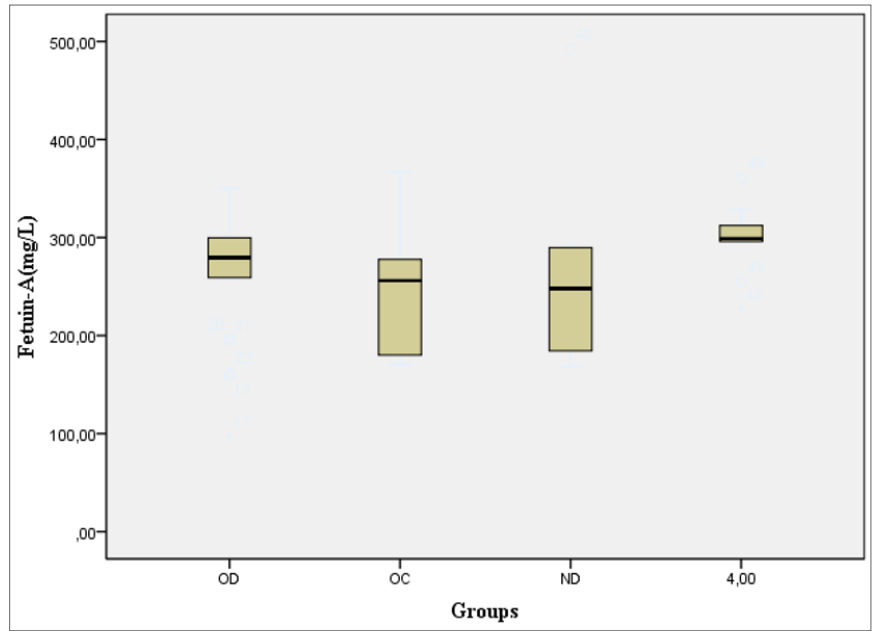

Figure 3. A Boxplot illustration of serum fetuin-A concentrations in subjects of overweight and normalweight diabetic patients and their healty controls. OD: overweight diabetic, OC: overweight control, ND: normalweight diabetic, NC: normalweight control

Table 1. Demographic and laboratory datas of overweight and normalweight diabetic patients and their controls.

\begin{tabular}{|lccccccccc|} 
& \multicolumn{2}{c}{ OD $(\mathbf{n}=\mathbf{2 3})$} & \multicolumn{2}{c}{ OC $(\mathbf{n}=\mathbf{1 1})$} & \multicolumn{2}{c}{ ND $(\mathbf{n}=7)$} & \multicolumn{2}{c}{ NC $(\mathbf{n}=\mathbf{9})$} \\
\cline { 2 - 9 } & Median & Min-Max & Median & Min-Max & Median & Min-Max & Median & Min-Max \\
\hline Age (years) & 61 & $54-67$ & 59 & $53-64$ & 55 & $53-62$ & 58 & $51-65$ \\
Height $(\mathrm{cm})$ & 163 & $150-188$ & 170 & $159-193$ & 166 & $150-172$ & 165 & $158-182$ \\
Weight $(\mathrm{kg})$ & $81.5 \mathrm{~b}$ & $75.4-111$ & 78 & $76-116.5$ & 62.4 & $47.7-66$ & 65 & $50.8-71.3$ \\
BMI $\left(\mathrm{kg} / \mathrm{m}^{2}\right)$ & $28.91 \mathrm{~b}$ & $26.15-29.33$ & 27.96 & $26.10-29.93$ & 22.57 & $20.89-23.57$ & 23.67 & $21.03-24.98$ \\
FBG $(\mathrm{mg} / \mathrm{dl})$ & $137^{* *}$ & $117-162$ & 98 & $88-110$ & $140^{*}$ & $101-164$ & 91 & $82-101$ \\
Fasting insülin $(\mu \mathrm{U} / \mathrm{ml})$ & $12.65 \mathrm{a}$ & $8.56-17.40$ & 12.20 & $9.20-14.50$ & $6.05^{*}$ & $4.40-7.52$ & 10.20 & $7.40-12.38$ \\
HOMA-IR & $5.15 \mathrm{a}$ & $4.36-6.81$ & 4.01 & $3.21-4.28$ & 2.12 & $2.07-3.13$ & 2.18 & $1.62-3.58$ \\
HbAlc $(\%)$ & $6.75^{* *}$ & $5.30-8.15$ & 5.30 & $4.20-6.00$ & $6.51^{* *}$ & $5.83-7.60$ & 5.20 & $4.40-5.67$ \\
\hline
\end{tabular}




\begin{tabular}{|c|c|c|c|c|c|c|c|c|c|c|c|c|}
\hline & \multicolumn{3}{|c|}{ OD $(n=23)$} & \multicolumn{3}{|c|}{$\mathrm{OC}(\mathrm{n}=11)$} & \multicolumn{3}{|c|}{$\mathrm{ND}(\mathrm{n}=7)$} & \multicolumn{3}{|c|}{$\mathrm{NC}(\mathrm{n}=9)$} \\
\hline & Visfatin & Eotaxin & Fetuin-A & Visfatin & Eotaxin & Fetuin-A & Visfatin & Eotaxin & Fetuin-A & Visfatin & Eotaxin & Fetuin-A \\
\hline Visfatin (ng/ml) & - & 0.003 & -0.284 & - & $0.700^{*}$ & 0.291 & - & 0.714 & 0.657 & - & -0.377 & 0.226 \\
\hline Eotaxin (ng/L) & 0.003 & - & 0.315 & $0.700^{*}$ & - & 0.291 & 0.714 & - & 0.714 & -0.377 & - & 0.183 \\
\hline Fetuin-A (mg/L) & -0.284 & 0.315 & - & 0.291 & 0.291 & - & 0.657 & 0.714 & - & 0.226 & 0.183 & - \\
\hline BMI $\left(\mathrm{kg} / \mathrm{m}^{2}\right)$ & $0.474^{*}$ & 0.038 & -0.292 & $0.782^{\star *}$ & 0.518 & .000 & 0.257 & 0.429 & -0.086 & $-0.695^{\star}$ & 0.283 & -0.133 \\
\hline FBG (mg/dl) & 0.179 & -0.336 & -0.394 & $0.609^{*}$ & 0.455 & 0.255 & -0.771 & $-0.943^{\star *}$ & -0.771 & -0.017 & -0.360 & -0.184 \\
\hline Fasting insulin $(\mu \mathrm{U} / \mathrm{ml})$ & 0.192 & 0.293 & 0.364 & $0.709^{*}$ & 0.445 & 0.345 & -0.029 & -0.200 & -0.029 & 0.544 & -0.067 & $0.817^{\star *}$ \\
\hline HbA1c (\%) & 0.231 & 0.017 & -0.234 & -0.148 & -0.491 & -0.343 & -0.696 & $-0.899^{*}$ & -0.696 & -0.658 & 0.281 & 0.026 \\
\hline HOMA-IR & 0,252 & 0,220 & 0,241 & $0,691^{*}$ & 0,391 & 0,309 & $-0,058$ & $-0,319$ & $-0,145$ & $0,703^{\star}$ & $-0,233$ & $0,667^{*}$ \\
\hline
\end{tabular}

As shown in Table 2. A positive correlation was found between the visfatin level and BMI in OD and $\mathrm{OC}(\mathrm{p}<0.05$ and $\mathrm{p}<0.01$ ), while a negative correlation was found in NC. A linear correlation was determined between visfatin level and eotaxin, FBG, fasting insulin level, and HOMA-IR in OC $(\mathrm{p}<0.05)$. A positive correlation was determined between visfatin level and HOMA-IR in the NC $(\mathrm{p}<0.05)$. A negative correlation was observed between the eotaxin levels and FBG and HbA1c levels in the ND $(\mathrm{p}<0.05$ and $\mathrm{p}<0.01)$, while a positive correlation was determined between the fetuin-A levels and fasting insulin levels and insulin resistance in the $\mathrm{NC}(\mathrm{p}<0.01$ and $\mathrm{p}<0.05)$.

\section{DISCUSSION}

Owerweight and/or obesity is a major risk factor of T2DM, is closely related to irregular adipokine release, macrophage infiltration into adipose tissue and inflammation processes. Very little is known about the potential role of these processes in the development of T2DM. This study examined the serum visfatin, eotaxin and fetuin-A levels in normal and owerweight patients with T2DM with the purpose of contributing to closing the gap regarding this issue.

Individuals with T2DM may be exposed to various complications despite receiving medical treatment. In cases where medical treatment fails to satisfy, hyperglycemia, increased $\mathrm{HbA} 1 \mathrm{c}$ and increased insulin resistance can be observed. In the present study, FBG levels and HbA1c concentrations were significantly higher in the normal and overweight patients with T2DM who received oral antidiabetic treatment. The fasting insulin level and insulin resistance of the OD were higher than the ND. Similarly, these parameters were determined to be higher in the OC compared to the NC. In general, insulin resistance is high in patients with T2DM and is more exacerbated in the presence of obesity (17). The acquired data were in support of the literature indicating that there were relationships between fasting insulin level, insulin resistance and $\operatorname{BMI}(4,5,18,19)$. Despite there being unsurprising and expected results, it was observed that the participants of the present study were not fully biochemically controlled with the antidiabetic treatment they were receiving. This may be due to them not using their medication regularly, not paying attention to their diet and periodic health checks, and their sedentary lifestyle habits.

Adipose tissue, which was known only as an energy store until approximately 10-15 years ago, is now known as the largest endocrine organ in the human body. As an active tissue, the adipose tissue, secretes important proteins called adipokine that have metabolic activity. Some of the adipokines secreted from this tissue may be protective against T2DM. One of these is visfatin, which acts like insulin in muscles, adipose tissue, and liver and increases insulin sensitivity (9). The antidiabetic effects of visfatin, insulin resistance and its relationship with T2DM have been investigated in many studies. However, contradictory results have been obtained in similar studies conducted on this subject. Some researchers have reported that there is no relationship between plasma visfatin level and diabetes or vice versa. Takebayashi et al. (20) determined that there was no significant relationship between visfatin level and T2DM. Gündüz et al. (21) found that the visfatin levels of the control group and patients with T2DM were similar. Toruner et al. (22) reported that plasma visfatin levels were significantly lower in T2DM patients. Moreover, they determined that there was a negative relationship between visfatin and $\mathrm{HbA1c}$ levels. Contrary to the findings of previous studies, the present study found that visfatin levels of the patients with T2DM were higher compared to those of the control group. Similarly, in a study conducted with a large number of patients it was found that visfatin concentrations were significantly higher in T2DM patients compared to the control group (23). Lopez et al. (24) determined that plasma visfatin levels were positively related with insulin resistance and that visfatin may be significant in the pathogenesis of diabetes. Habib et al. (25) found the serum visfatin levels of patients with T2DM to be $7.01 \pm 3.79 \mathrm{ng} / \mathrm{ml}$ and those of healthy subjects to be $4.02 \pm 2.74 \mathrm{ng} / \mathrm{ml}(\mathrm{p}=0.046)$. 
Researchers have found that patients with T2DM have high visfatin levels and higher visfatin levels with poor glycemic control and increased body adiposity indices. Haider et al. (26) determined that when blood glucose increased plasma visfatin levels also increased in healthy participants who underwent glucose infusion tests. In addition, they reported that when the glucose level increased in fat cell cultures, the expression of visfatin also increased. With this regard, the results of the present study agreed with these results and those of various other studies in the literature $(27,28,29)$. When the findings of the present study and previous studies are considered, it was considered that this increase in visfatin levels in the T2DM patients was likely to be due to the impairment of the visfatin signal in target tissues, the development of visfatin resistance or an increase in biosynthesis due to hyperglycemia, hyperinsulinemia, or the reaction to adipokines, and was accepted as a protective physiological mechanism (29).

In this study, a positive correlation was determined between visfatin levels and BMI. Similarly, in the relevant studies conducted in the literature a significant positive correlation was also reported between visfatin levels and BMI (27, 30-32). Choi et al. (33) determined that visfatin levels of non-obese patients were lower than obese patients. Moreover, they found that the plasma visfatin levels could be decreased when body weight was reduced by means of an exercise program. Kara et al. (32) compared obese and non-obese patients with T2DM with healthy control groups and determined that the serum visfatin levels of obese patients with T2DM were higher compared to those of the control groups.

In brief, the association between serum visfatin levels and diabetes has not been fully explained with the studies conducted thus far. According to the results of this study, the visfatin levels were significantly higher in the OD and ND. However, visfatin levels were also determined to be high in the OC. The findings of this study proved that there was a notable association between visfatin and obesity and/or visfatin and diabetes. However, the role of visfatin in the pathophysiology of diabetes is still controversial. Although it is believed that visfatin may be a compensatory mechanism in this process, the role of visfatin in insulin resistance related disorders is not fully known.

It has become increasingly clear that diabetes is a lowgrade inflammatory disease, and that inflammation is closely related to insulin resistance (34). In the presence of T2DM, changes can be observed in the serum levels of inflammatory markers. Determining inflammatory markers in diabetes can be important in gaining insight into the processes underlying the onset and progression of the disease. A clearer understanding of the inflammatory basis of T2DM may also be significant in putting forward new approaches to currently used pharmacological/ non-pharmacological interventions (35). Studies on biomarkers associated with the chronic inflammatory condition underlying T2DM and their interactions with one another are ongoing (36-39).

Eotaxin, which is an important pro-inflammatory cytokine is synthesized in various cells of the immune system. It has also been determined that it is secretory product of adipose tissue and that plasma levels are increased in obese individuals (11). Vasudevan et al. (11) determined that serum eotaxin level in obese individuals were high. However, in the present study, it was determined that the serum eotaxin levels of obese and non-obese participants were similar. As eotaxin is a pro-inflammatory agent and has previously been reported to have a possible role in the development of obesity-related disorders such as T2DM, its associated with T2DM was determined. In the literature review, only one study was found to have directly examined the relationship between T2DM and eotaxin. In this study, conducted by Herder et al. (40) no changes were determined in the serum eotaxin levels of patients with T2DM. However, in the present study, it was observed that serum eotaxin levels were higher in OD and ND compared to their control groups. These results suggest that eotaxin may play a part in glucose metabolism and the pathogenesis of T2DM.

Fetuin-A is a multifunctional molecule that is secreted from the liver. According to the results of previous studies, fetuin-A is a biomarker of the risk of T2DM $(14,41)$. Ix et al. (14) reported that among the healthy individuals they followed up for 6 years the serum fetuin-A levels of 135 patients who developed diabetes were higher than those of the healthy individuals who did not develop diabetes. Furthermore, they determined that there was a relationship between serum fetuin-A levels and insulin resistance. Song et al. (42) determined that middle-aged subjects with T2DM showed high fetuin-A levels and that there was a positive correlation between the fetuin-A level and insulin resistance. However, some researchers have reported no significant difference between fetuin-A levels between patients with T2DM and healthy individuals, and no relationship between fetuin-A levels and insulin resistance $(12,21)$. Yllmaz et al. (43) determined that fetuin-A levels were significantly lower in T2DM patients. In the present study, no significant difference was observed in serum fetuin-A levels between the OD and ND patients with T2DM and their control groups. However, we determined a positive relationship between the serum fetuin-A and fasting insulin levels and insulin resistance in the NC. These findings are consistent with the results of various 
similar studies $(12,21)$, while they contradict the results of others $(41,44)$. The differences between the results may be due to the subjects being of different ethnic origins and gender, having different lifestyles, and dietary habits and receiving different medication.

Our study has certain limitations; firstly the metabolic parameters of the T2DM patients included in the study were not under control. Second, the small number of patients in the study. Third, It can be listed as the fact that the compliance of patients with drug therapy was not evaluated.

\section{CONCLUSION}

Serum visfatin and eotaxin levels are high in patients with T2DM, and this elevation is dependent of BMI. In addition, visfatin level is high in overweight non-diabetic subjects.

\section{ETHICAL DECLARATION}

Ethics Committee Approval: The study was approved by the Non-interventional Studies Ethics Committee of Dicle University Faculty of Medicine (Date: 15.04.2015, Decision No: 195).

Informed Consent: All patients signed the free and informed consent form.

\section{Referee Evaluation Process: Externally peer-reviewed.}

Conflict of Interest Statement: The authors have no conflicts of interest to declare.

Financial Disclosure: The authors would like to thank the Dicle University Scientific Research Project Coordinator (DÜBAP) for its financial support for this research.

Author Contributions: All of the authors declare that they have all participated in the design, execution, and analysis of the paper, and that they have approved the final version.

Note: A part of this study was presented as a poster presentation at the 43rd National Physiology Congress in Turkey (07 - 10 September 2017, Denizli).

\section{REFERENCES}

1. American Diabetes Association. Standards of medical care in diabetes-2015. Diabetes Care 2015; 38: S1-S2.

2. Mitrakou A, Kelley D, Mokan M, et al. Role of reduced suppression of glucose production and diminished early insulin release in impaired glucose tolerance. N Engl J Med 1992; 326: 22-9.

3. Reaven GM. Banting lecture 1988. Role of insulin resistance in human disease. Diabetes 1988; 37: 1595-607.

4. Chen L, Magliano DJ, Zimmet PZ. The worldwide epidemiology of type 2 diabetes mellitus: present and future perspectives. Nat Rev Endocrinol 2011; 8; 8: 228-36.
5. Hu FB. Globalization of diabetes: the role of diet, lifestyle, and genes. Diabetes Care 2011; 34: 1249-57.

6. Opara E. Nutrition and diabetes. Pathophysiology and management. Taylor and Francis Group 2006.

7. Centers for Disease Control and Prevention (CDC). Prevalence of overweight and obesity among adults with diagnosed diabetes-United States, 1988-1994 and 1999-2002. Morb Mortal Wkly Rep. (MMWR) 2004; 19; 53: 1066-8.

8. Xu H, Barnes GT, Yang Q, et al. Chronic inflammation in fat plays a crucial role in the development of obesity-related insulin resistance. J. Clin. Invest 2003; 112: 1821-30.

9. Fukuhara A, Matsuda M, Nishizawa M, et al. Visfatin: a protein secreted by visceral fat that mimics the effects of insulin. Science 2005 Jan 21; 307: 426-30.

10. Jean-Baptiste S, O’Toole EA, Chen M, Guitart J, Paller A, Chan LS. Expression of eotaxin, an eosinophil-selective chemokine, parallels eosinophil accumulation in the vesiculobullous stage of incontinentia pigmenti. Clin Exp Immunol 2002; 127: 470-8.

11. Vasudevan AR, Wu H, Xydakis AM, et al. Eotaxin and obesity. J Clin Endocrinol Metab 2006; 91: 256-61.

12. Mori K, Emoto M, Yokoyama H, et al. Association of serum fetuin-A with insulin resistance in type 2 diabetic and nondiabetic subjects. Diabetes Care 2006; 29: 468.

13. Ix JH, Shlipak MG, Brandenburg VM, Ali S, Ketteler M, Whooley MA. Association between human fetuin-A and the metabolic syndrome: data from the Heart and Soul Study. Circulation 2006; 113: 1760-7.

14. Ix JH, Wassel CL, Kanaya AM, et al. Fetuin-A and incident diabetes mellitus in older persons. JAMA 2008; 300: 182-8.

15. Mathews ST, Rakhade S, Zhou X, Parker GC, Coscina DV, Grunberger G. Fetuin-null mice are protected against obesity and insulin resistance associated with aging. Biochem Biophys Res Commun 2006; 350: 437-43.

16. Matthews DR, Hosker JP, Rudenski AS, Naylor BA, Treacher DF, Turner RC. Homeostasis model assessment: insulin resistance and beta-cell function from fasting plasma glucose and insulin concentrations in man. Diabetologia 1985; 28: 412-9.

17. Kelley DE, Williams KV, Price JC, McKolanis TM, Goodpaster $\mathrm{BH}$, Thaete FL. Plasma fatty acids, adiposity, and variance of skeletal muscle insulin resistance in type 2 diabetes mellitus. J Clin Endocrinol Metab 2001; 86: 5412-9.

18. Ruderman N, Chisholm D, Pi-Sunyer X, Schneider S. The metabolically obese, normal-weight individual revisited. Diabetes 1998; 47: 699-713.

19. Huxley R, James WP, Barzi F, et al. Ethnic comparisons of the cross-sectional relationships between measures of body size with diabetes and hypertension. Obes Rev 2008 Mar; 9: 53-61.

20. Takebayashi K, Suetsugu M, Wakabayashi S, Aso Y, Inukai T. Association between plasma visfatin and vascular endothelial function in patients with type 2 diabetes mellitus. Metabolism 2007; 56: 451-8.

21. Gunduz FO, Yildirmak ST, Temizel M, et al. Serum visfatin and fetuin-a levels and glycemic control in patients with obese type 2 diabetes mellitus. Diabetes Metab J 2011; 35: 523-8.

22. Toruner F, Altinova AE, Bukan N, et al. Plasma visfatin concentrations in subjects with type 1 diabetes mellitus. Horm Res 2009; 72: 33-7.

23. Sandeep S, Velmurugan K, Deepa R, Mohan V. Serum visfatin in relation to visceral fat, obesity, and type 2 diabetes mellitus in Asian Indians. Metabolism 2007; 56: 565-70.

24. López-Bermejo A, Chico-Julià B, Fernàndez-Balsells $\mathrm{M}$, et al. Serum visfatin increases with progressive beta-cell deterioration. Diabetes 2006; 55: 2871-5.

25. Habib SS, Bashir S, Habib SH. Serum visfatin relationship with glycemic control and adiposity indices in patients with type 2 diabetes mellitus. Khyber Med Univ J 2020; 12: 263-7. 
26. Haider DG, Schaller G, Kapiotis S, Maier C, Luger A, Wolzt M. The release of the adipocytokine visfatin is regulated by glucose and insulin. Diabetologia 2006; 49: 1909-14.

27. El-Shaer OS, Belal KM, Issa HA, El-Adl T. Increased serum visfatin levels in patients with type 2 diabetic patients. Life Sci. J 2012; 9: 114-20.

28. Dogru T, Sonmez A, Tasci I, et al. Plasma visfatin levels in patients with newly diagnosed and untreated type 2 diabetes mellitus and impaired glucose tolerance. Diabetes Res Clin Pract 2007; 76: 24-9.

29. Chen MP, Chung FM, Chang DM, et al. Elevated plasma level of visfatin/pre-B cell colony-enhancing factor in patients with type 2 diabetes mellitus. J Clin Endocrinol Metab 2006; 91: 295-9.

30. Varma V, Yao-Borengasser A, Rasouli N, et al. Human visfatin expression: relationship to insulin sensitivity, intramyocellular lipids, and inflammation. J Clin Endocrinol Metab 2007; 92: 66672.

31. Berndt J, Klöting N, Kralisch S, et al. Plasma visfatin concentrations and fat depot-specific mRNA expression in humans. Diabetes 2005; 54: 2911-6.

32. Kara M, Uslu S, Kebapçı N, Özçelik E, Bal C. Evaluation of the serum visfatin and adiponectin levels in patients with type 2 diabetes mellitus. Turkish J. Biochem 2014; 39: 181-7.

33. Choi KM, Kim JH, Cho GJ, Baik SH, Park HS, Kim SM. Effect of exercise training on plasma visfatin and eotaxin levels. Eur J Endocrinol 2007; 157: 437-42.

34. Esposito K, Giugliano D. The metabolic syndrome and inflammation: association or causation? Nutr Metab Cardiovasc Dis 2004 ; $14: 228-32$

35. King GL. The role of inflammatory cytokines in diabetes and its complications. J Periodontol 2008; 79: 1527-34.

36. Pickup JC. Inflammation and activated innate immunity in the pathogenesis of type 2 diabetes. Diabetes Care 2004; 27: 813-23.

37. Lukic L, Lalic NM, Rajkovic N, et al. Hypertension in obese type 2 diabetes patients is associated with increases in insulin resistance and IL-6 cytokine levels: potential targets for an efficient preventive intervention. Int J Environ Res Public Health 2014; 11: 3586-98.

38. Kashiwagi R, Yamada Y, Ito Y, et al. Increase in adiponectin level prevents the development of type 2 diabetes in japanese men with low adiponectin levels. J Endocr Soc 2018; 2: 753-64.

39. Zheng $\mathrm{M}$, Wang $\mathrm{X}$, Guo $\mathrm{H}$, et al. The cytokine profiles and immune response are increased in covid-19 patients with type 2 diabetes mellitus. J Diabetes Res 2021; 2021: 9526701.

40. Herder C, Haastert B, Müller-Scholze S, et al. Association of systemic chemokine concentrations with impaired glucose tolerance and type 2 diabetes: results from the Cooperative Health Research in the Region of Augsburg Survey S4 (KORA S4). Diabetes 2005; 54: S11-7.

41. Sun Q, Cornelis MC, Manson JE, Hu FB. Plasma levels of fetuin-A and hepatic enzymes and risk of type 2 diabetes in women in the U.S. Diabetes 2013; 62: 49-55.

42. Song A, Xu M, Bi Y, et al. Serum fetuin-A associates with type 2 diabetes and insulin resistance in Chinese adults. PLoS One 2011; 6: e19228.

43. Yilmaz MI, Saglam M, Qureshi AR, et al. Endothelial dysfunction in type-2 diabetics with early diabetic nephropathy is associated with low circulating adiponectin. Nephrol Dial Transplant 2008; 23: $1621-7$.

44. Dutta D, Mondal SA, Kumar M, et al. Serum fetuin-A concentration predicts glycaemic outcomes in people with prediabetes: a prospective study from eastern India. Diabet Med 2014 Dec; 31: 1594-9 